\title{
Controle Extremal Cíclico Multivariável via Função de Monitoração *
}

\author{
Nerito Oliveira Aminde* Tiago Roux Oliveira ${ }^{* *}$ Liu Hsu ${ }^{*}$ \\ * Programa de Eng. Elétrica, COPPE/UFRJ, Rio de Janeiro, Brasil, \\ (e-mails:nerito@coep.ufrj.br,liu@coep.ufrj.br). \\ ** Departamento de Eng. Eletrônica e Telecomunicações, \\ DETEL/UERJ, Rio de Janeiro, Brasil (e-mail: tiagoroux@uerj.br)
}

\begin{abstract}
This paper addresses the design of a cyclic extremum-seeking controller based on monitoring function for a class of non-linear multivariable static systems. We demonstrate that it is possible to achieve an arbitrarily small neighborhood of the desired optimal point (extremum) from all initial conditions, i.e., the algorithm has global convergence properties. To achieve such goal, the controller tries to perform the best search on each direction, cyclically. Simulation results with a two-input system and one single output illustrate significant advantages of the proposed multivariable control strategy in terms of fast convergence and small residual errors.

Resumo: Este artigo apresenta o projeto de um controlador extremal cíclico baseado em função de monitoração para uma classe de sistemas não-lineares estáticos multivariáveis. Demonstra-se que é possível alcançar uma vizinhança arbitrariamente pequena do extremo ótimo partindo-se de quaisquer condições iniciais, isto é, o algoritmo tem propriedades globais de convergência. Para isso, o controlador tenta fazer a melhor busca numa direção de cada vez, ciclicamente. Resultados de simulação com um sistema de duas entradas e uma única saída ilustram as vantagens significativas da estratégia de controle multivariável proposta, em termos de rapidez de convergência e de erros residuais pequenos.
\end{abstract}

Keywords: Adaptive Control; Extremum Seeking Control; Multivariable Nonlinear Systems, Monitoring Function; Cyclic search direction

Palavras-chaves: Controle Adaptativo; Controle Extremal; Sistemas Não-lineares Multivariáveis; Função de Monitoração; Direção de busca ciclica.

\section{INTRODUÇÃO}

Controle por busca extremal ou simplesmente, controle extremal (do inglês, Extremum Seeking Control - ESC) é um metodo de controle que procura levar os estados de um sistema para um ponto que otimiza um dado índice de desempenho. A função objetivo é completa ou parcialmente desconhecida, devido às incertezas no sistema (Tan et al., 2010; Yaghoubi et al., 2018)). Este método lida com o problema de otimização em tempo real, podendo o mapeamento não-linear ser escalar ou multivariável. Uma abordagem multivariável detalhada pode ser encontrada em Rotea (2000); Krstić and Wang (2000); Ariyur and Krstić $(2002,2003)$.

Em Tan et al. (2010), pode-se encontrar uma série de aplicações de ESC, entre elas, o projeto de sistemas de freios ABS, veículos autônomos e robôs móveis, motores a combustão interna, controle de processos e até aceleradores de partículas.

A maioria das publicações na literatura está concentrada em sistemas de uma entrada e uma saída (SISO). Entre-

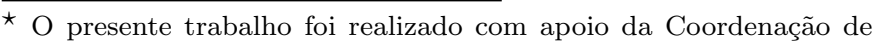
Aperfeiçoamento de Pessoal de Nível Superior Brasil (CAPES) Código de Financiamento 001, CNPq, FAPERJ, ISDB-Maputo e a CINOP Global.
}

tanto, muitos problemas que requerem otimização são multivariáveis (Ariyur and Krstić, 2003). Por isso, nos últimos anos, várias técnicas de controle extremal multivariável foram propostas (Ariyur and Krstić, 2002; Ghaffari et al., 2012; Toloue and Moallem, 2017; Salamah and Özguner, 2018). Essas técnicas de controle podem ser aplicadas a mapeamentos acoplados entre os canais de entrada (Xiao et al., 2014) ou desacoplados (Toloue and Moallem, 2017; Salamah and Özguner, 2018). Uma desvantagem do controle de mapeamento desacoplado é a falta de interação entre os canais de entrada (Toloue and Moallem, 2016), por isso, nesse artigo será considerado o caso em que há um mapeamento acoplado.

De acordo com Calli et al. (2012), o controle extremal pode ser dividido em cinco categorias, ESC baseado em perturbação periódica ou dithers, ESC baseado por modos deslizantes, ESC baseado em redes neurais, ESC baseado por aproximação linear e ESC adaptativo, sendo o ESC baseado em perturbação, o mais popular (Krstić and Wang, 2000). Versões de ESC por modos deslizantes com funções de chaveamento periódica são encontradas em Oliveira et al. (2011, 2012); Toloue and Moallem (2016).

Recentemente, foi proposta uma nova estratégia de controle extremal (Aminde et al., 2013; Hsu et al., 2014) baseada em funções de monitoração (Yan et al., 2003, 2006; 
Oliveira et al., 2010). Embora os resultados para plantas de grau relativo um em Aminde et al. (2013) tenham sido generalizados para grau relativo arbitrário (e desconhecido) em Oliveira et al. (2014), ambas as publicações consideraram o caso SISO.

No recente artigo dos autores (Aminde et al., 2018), apresentou-se uma extensão dos resultados escalares alcançados anteriormente para o caso multivariável, mais especificamente o caso de múltiplas entradas e única saída (MISO). Com a existência de acoplamento entre os canais de entrada, o esquema de controle é mais complexo, uma vez que além de $n$ leis de controle, são necessárias $n$ funções de monitoração, $n$ modelos de referências, etc. No presente artigo, apresenta-se um controlador cíclico via função de monitoração, inspirados em Salamah and Özguner (2018), que basicamente trata o sistema multivariável como uma sequência de sistemas escalares, o que resulta na redução substancial de complexidade no esquema de controle. A utilização de função de monitoração parece vantajosa por permitir rápida convergência em relação ao artigo citado que se baseia em modos deslizantes e função de chaveamento periódica. Simulações numéricas que ilustram a aplicabilidade do método são apresentadas.

Notação e Terminologia - Ao longo do artigo, a norma Euclidiana de um vetor $x$ e a norma induzida da matriz $A$ correspondente são denotadas como $\|x\|$ and $\|A\|$, respectivamente. Do ponto de vista técnico, os resultados teóricos obtidos neste artigo são baseados na definição de solução de Fillipov para equações diferenciais com lado direito descontínuo (Filippov, 1964).

\section{FORMULAÇÃO DO PROBLEMA}

Considere um mapeamento multivariável não-linear $h$ : $\mathbb{R}^{n} \rightarrow \mathbb{R}$ dado por

$$
y=h(x),
$$

onde $x \in \mathbb{R}^{n}$ e $y \in \mathbb{R}$. Deseja-se encontrar o vetor $x^{*}$ que, sem perda de generalização, maximiza a saída $y$. Supõe-se que tal ponto de máximo $y^{*}=h\left(x^{*}\right)$ é único. Esse problema pode ser formulado no contexto de controle extremal de um sistema de controle em que $y$ é a saída e $x$ é visto como sendo a saída de integrador cuja entrada é dada por um vetor $v$ de sinais de controle a serem projetados. Assim, considera-se o seguinte sistema

$$
\begin{aligned}
& \dot{x}=v \\
& y=h(x)
\end{aligned}
$$

onde $v \in \mathbb{R}^{n}$ é vetor de entrada e $x=\left[x_{1} \cdots x_{n}\right]^{T}$. De modo a garantir existência e unicidade de soluções, a função não-linear $h(x)$ é localmente Lipschitz contínua em $x$ e suficientemente suave (todas as derivadas são contínuas). Para cada solução de (2)-(3), existe intervalo de tempo máximo de definição dado por $\left[0, t_{M}\right)$, onde $t_{M}$ pode ser finito ou infinito.

O controle extremal pode ser reformulado como um problema de rastreamento em que a direção de controle é desconhecida (Oliveira et al., 2012). Portanto, deseja-se encontrar uma lei de controle $v$ (vectorial) por realimentação de saída tal que, a partir de quaisquer condições iniciais, o sistema é conduzido para alcançar o ponto extremo $y^{*} \mathrm{e}$ permanecer o mais próximo possível do mesmo.
Cabe notar que sistemas mais gerais podem ser considerados (Aminde et al., 2013; Oliveira et al., 2014), nos quais $x$ seria a saída de um sistema de controle com dinâmica mais complexa do que a de simples integrador. O objetivo de considerar-se o sistema (2)-(3) é de focar no método aqui proposto para busca extremal em sistemas dinâmicos em que a função objetivo a ser otimizada depende de várias entradas.

De (2) e (3), a derivada da saída $y$ em relação ao tempo é dada por

$$
\dot{y}=\frac{\partial h^{T}}{\partial x} v
$$

onde o ganho de alta frequência (do inglês, High Frequence Gain - HFG) é dado pelo vetor gradiente, i.e.,

$$
\begin{gathered}
k_{p}(x):=\left[\begin{array}{lll}
k_{p_{1}} & \cdots & k_{p_{n}}
\end{array}\right] \\
k_{p}(x):=\frac{\partial h^{T}}{\partial x}=\left[\begin{array}{lll}
\frac{\partial h}{\partial x_{1}} & \cdots & \frac{\partial h}{\partial x_{n}}
\end{array}\right] .
\end{gathered}
$$

Do mesmo modo que em Oliveira et al. (2012), os sinais dos elementos $k_{p_{i}}:=\frac{\partial h}{\partial x_{i}}$ de $k_{p}$, podem ser vistos como as direções de controle, sendo (5) e (6) equivalentes.

Com relação à planta controlada, assumem-se as seguintes hipóteses:

(H1) (Diferenciabilidade de $h$ ): A função $h: \mathbb{R}^{n} \rightarrow \mathbb{R}$ é continuamente duplamente diferenciável em todo o $\mathbb{R}^{n}$.

(H2) (Extremo máximo único): Existe um único vetor $x^{*} \in \mathbb{R}^{n}$ tal que $y^{*}=h\left(x^{*}\right)$ é um extremo máximo de $h(x): \mathbb{R}^{n} \rightarrow \mathbb{R}$ no qual o gradiente $\frac{\partial h}{\partial x}=0$, e para todo $\Delta>0$, suficientemente pequeno, existe uma constante $L_{h}(\Delta)>0$, tal que

$$
L_{h}(\Delta) \leq\left\|\frac{\partial h}{\partial x}\right\|, \quad \forall x \notin \mathcal{D}_{\Delta}, i=1, \ldots, n,
$$

sendo $\mathcal{D}_{\Delta}:=\left\{x:\left\|x-x^{*}\right\|<\Delta / 2\right\}$ e $\Delta$ pode ser feito arbitrariamente pequeno, bastando permitir que $L_{h}$ seja suficientemente pequeno, em virtude da hipótese (H1) .

A busca do extremo de $h$, leva à consideração de um sistema de controle não-linear com HFG vetorial dependente do estado, que muda de direção em torno do ponto extremo de modo contínuo. No problema de busca extremal, supõese que apenas o valor instantâneo de $h$ é conhecido mas que o seu gradiente instantâneo é desconhecido.

De $(6)$ e $(\mathbf{H 2}), k_{p}$ satisfaz $\left(\forall x_{i} \notin \mathcal{D}_{\Delta_{i}}\right)$ :

$$
0<\underline{k}_{p} \leq\left\|k_{p}\right\|
$$

onde o limitante inferior $\underline{k}_{p} \leq L_{h}$ é uma constante.

\section{CONTROLADOR EXTREMAL CÍCLICO MULTIVARIÁVEL VIA FUNÇÃO DE MONITORAÇÃO}

O esquema de controle extremal cíclico multivariável via função de monitoração proposto está representado na Figura 1. A lei de controle para plantas com HFG desconhecido é definida de forma similar a Oliveira et al. (2007): 


$$
v(t)=\left\{\begin{array}{l}
v(t)^{+}=-\rho(t) \sigma(t) \operatorname{sgn}(e(t)), t \in T^{+}, \\
v(t)^{-}=\rho(t) \sigma(t) \operatorname{sgn}(e(t)), \quad t \in T^{-},
\end{array}\right.
$$

onde $\rho(t)$ é função de modulação a ser projetada, $\sigma(t)$ é a função de busca cíclica a ser definida, responsável por escolher, periodicamente, a direção de busca, os conjuntos $T^{+}$e $T^{-}$satisfazem $T^{+} \cap T^{-}=\emptyset$ e $T^{+} \cup T^{-}=\left[0, t_{M}\right)$.

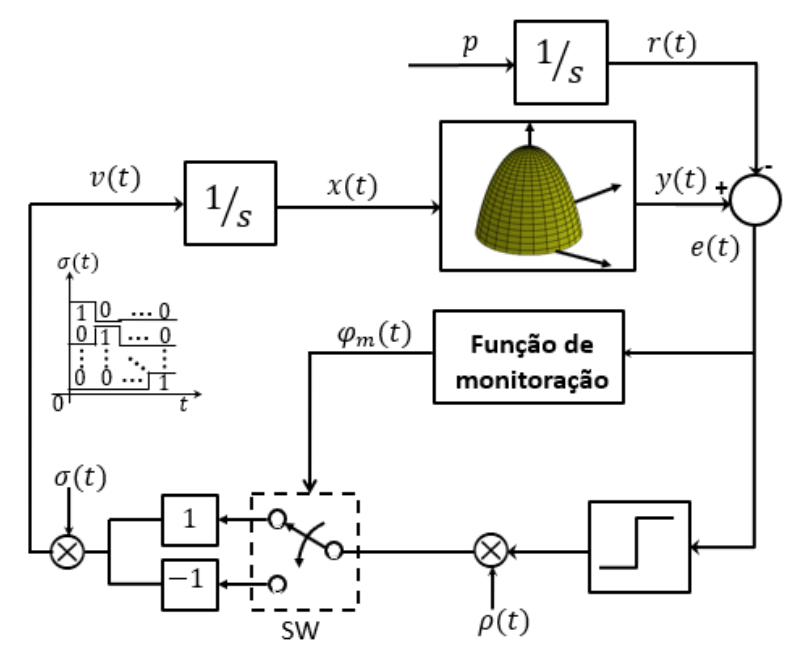

Figura 1. Diagrama de controle extremal cíclico de sistemas multivariáveis proposto.

O sinal do erro $e(t)$ é definido como

$$
e(t)=y(t)-r(t)
$$

onde $r(t)$ é uma rampa crescente em função do tempo, gerada através da seguinte trajetória de referência

$$
\dot{r}(t)=p, r(0)=r_{0},
$$

onde $p>0, r_{0}$ são constantes de projeto. Para evitar sinal de referência ilimitado $r(t)$ no controlador, pode-se saturá-lo por um limitante superior de $y^{*}$ sem afetar o desempenho do controlador extremal.

A função de modulação $\rho(t)$ será projetada de forma que $y(t)$ possa rastrear o modelo de referência $r(t), \forall t$, até que o ponto extremo seja alcançado. Deste modo, $y(t)$ é forçado a alcançar a vizinhança do máximo $y^{*}=h\left(x^{*}\right)$ e permanecer próximo do valor ótimo, isto é, na vizinhança do vetor maximizador $x^{*}$. Em outras palavras, cada parâmetro $x_{i}$ é forçado a alcançar a vizinhança de $x_{i}^{*}$. A partir de um certo tempo finito, obviamente a referência deixa de ser rastreada pois $h(x)$ fica limitado superiormente pelo seu valor máximo.

Consequentemente, o rastreamento será interrompido. Mas, a vizinhança do máximo já terá sido alcançada conforme desejado. A estratégia de controle proposta garante que $y$ permanecerá próximo a $y^{*}, \forall t>t^{*}$, conforme será mostrado mais adiante, no Teorema 1. A taxa de convergência de $x$ para a região $\mathcal{D}_{\Delta}$ definida em (H2) é dependente da função de modulação $\rho(t)$.

\subsection{Funcionamento da busca cíclica}

A busca cíclica é projetada de modo que ocorra mudança de direção de busca, periodicamente. Seja $\sigma(t)$ uma função periódica com periodo $T_{s}$, ou seja, $\sigma\left(t+T_{s}\right)=\sigma(t)$ (ver detalhe na Figura 1). Define-se um intervalo de tempo $\mathcal{T}=\left[\mathcal{T}_{l}, \mathcal{T}_{u}\right)$ (aberto à direita para evitar ambiguidades) de período $T_{s}=\mathcal{T}_{u}-\mathcal{T}_{l}$ e seja o conjunto de instantes $\tau_{1}, \tau_{2}, \cdots, \tau_{n}$, onde $\mathcal{T}_{l}=\tau_{1}<\tau_{2}<\cdots \tau_{n}<\tau_{n+1}=\mathcal{T}_{u}$, sendo $\Delta \tau_{i}=\left[\tau_{i}, \tau_{i+1}\right), \forall i=1, \ldots, n$, os $n$ sub-intervalos dentro de cada ciclo. Seja também a base ortogonal $a_{1}, a_{2}, \cdots, a_{n} \in \mathbb{R}^{n}$ sendo $a_{i}^{T}=[0, \cdots 0,1,0 \cdots 0]$, com o elemento unitário na i-ésima posição do vetor. Então, a direção de busca cíclica em (8) pode ser definida como segue:

$$
\sigma(t)=a_{i} \quad \forall t \in \Delta \tau_{i}, \forall i=1, \ldots, n,
$$

Por simplicidade, escolhe-se a duração de cada subintervalo $\Delta \tau_{i}$ igual a $\frac{T_{s}}{n}, \forall i$. Assim, durante esse intervalo, o controlador faz a busca nessa direção antes de chavear para outra direção (Salamah and Özguner, 2018) no subintervalo seguinte, ou seja, o controlador cíclico multivariável funciona como um controlador escalar em cada subintervalo $\Delta \tau_{i}$.

\subsection{Dinâmica do Erro}

De (3), (9) e (10), somando e subtraindo $\lambda e$ à derivada de $e$ (omitindo-se $t$ ) tem-se:

$$
\dot{e}=\sum_{i=1}^{n} \frac{\partial h}{\partial x_{i}} v_{i}-p+\lambda e-\lambda e,
$$

sendo para i-ésima direção de busca,

$$
\dot{e}=-\lambda e+k_{p_{i}}\left(v_{i}+d_{e}\right),
$$

onde $\lambda>0$ é uma constante apropriada de projeto e

$$
d_{e}:=\left(k_{p_{i}}\right)^{-1}(-p+\lambda e) .
$$

Suponha-se que inicialmente em $t=\tau_{i}$, i.e., no início do iésimo sub-período, tenhamos controlabilidade do erro, com $k_{p_{i}} \geq L_{h}(\Delta)$ (ver (H2)). O termo $d_{e}$, visto como uma perturbação, é majorado em valor absoluto por:

$$
\bar{d}_{e}:=L_{h}^{-1}(p+\lambda|e|) \geq\left|d_{e}\right| .
$$

Baseando-se no método da função de monitoração proposto em Yan et al. (2003); Oliveira et al. (2010), pode-se propor para $i$-ésima direção de busca do sistema (2)-(3) a lei de controle por modos deslizantes

$$
v_{i}=-\rho U(t) \operatorname{sgn}(e),
$$

sendo a função de modulação $\rho$ dada por

$$
\rho=\bar{d}_{e}+\delta,
$$

sendo $U(t)=+1$ ou -1 , de acordo com o sinal estimado de $k_{p_{i}}$ no instante $t$.

Então, usando o Lema de Comparação (Filippov, 1964), tem-se que $\forall t \geq \tau_{1}, t \in \Delta \tau_{1}$, enquanto $\left|k_{p_{i}}\right|>L_{h}(\Delta)$, a ser referida como condição de controlabilidade,

$$
\left|e_{i}(t)\right| \leq \zeta_{i}(t), \quad \zeta_{i}(t):=\left|e_{i}\left(\tau_{1}\right)\right| e^{-\lambda\left(t-\tau_{1}\right)},
$$

No diagrama de controle ilustrado na Figura 1, $U$ corresponde à posição do chaveamento SW.

Com a lei de controle (16)-(17), a equação do erro (13) é limitada pelo estado $e$ de forma afim. Assim sendo, é fácil concluir que as soluções são definidas $\forall t$, i.e., $t_{M}=\infty$, pois podem crescer no máximo exponecialmente.

\subsection{Função de Monitoração}

A função de monitoração foi introduzida e desenvolvida em Yan et al. (2003); Oliveira et al. $(2007,2010)$ e foi 
aplicada ao problema de busca extremal em Aminde et al. (2013). Partindo-se do princípio de que a desigualdade (18) é garantida quando a direção de controle é correta, é natural usar $\zeta_{i}$ como referência para se decidir quando o chaveamento de $v_{i}$ em (8) ocorre de $v_{i}^{+}$para $v_{i}^{-}$(ou viceversa), isto é, o chaveamento ocorre apenas quando (18) é violada.

Uma modificação simples em relação a $\zeta_{i}$ é feita para garantir que a função de monitoração majore sempre o erro mesmo que inicialmente a direção de controle, dada por $U$, esteja errada. Considere-se a seguinte função para a i-ésima direção de busca

$$
\varphi_{k, i}(t)=\left|e_{i}\left(t_{k, i}\right)\right| e^{-\lambda\left(t-t_{k, i}\right)}+\mu, \quad k=1, \cdots, N_{i}
$$

onde $t_{1, i}=\tau_{i}$ (início do sub-intervalo) e $t_{k, i}$, para $k>1$, são os tempos de chaveamento de direção de controle, $\mu$ é constante positiva arbitrariamente pequena. Esses chaveamentos ocorrem quando o gráfico da função de monitoração encontra o gráfico do erro (ver Figura 2) e uma mudança de direção de controle é realizada. Múltipos chaveamentos ocorrem quando se atinge a região de perda de controlabilidade direcional, o que ocorre na vizinhança de um extremo direcional, i.e., quando $k_{p_{i}} \leq L_{h}$. Perto de um ponto extremo, a perda de controlabilidade pode resultar em múltiplas ultrapassagens por esse extremo. Assim, possivelmente ocorrem mudanças de direção de controle que são detectadas pela função de monitoração definida a seguir,

$$
\varphi_{m i}(t):=\varphi_{k, i}(t), \quad \forall t \in\left[t_{k, i}, t_{k+1, i}\right)
$$

Note-se que de (19) e (20), tem-se $\left|e_{i}(t)\right|<\left|\varphi_{k, i}(t)\right|$ em $t=t_{k, i}$, definido como o instante em que a função de monitoração $\varphi_{m i}(t)$ encontra $\left|e_{i}(t)\right|$, isto é,

$$
t_{k+1, i}:=\left\{\begin{array}{lc}
\min \left\{t>t_{k, i}:\left|e_{i}(t)\right|=\varphi_{k, i}(t)\right\}, & \text { se existir, } \\
\tau_{i}+\frac{\mathcal{T}_{s}}{n}, & \text { caso contrário } .
\end{array}\right.
$$

A seguinte desigualdade vem de (20)

$$
|e(t)| \leq \varphi_{m}(t), \quad \forall t \in\left[0, t_{M}\right),
$$

sendo $\varphi_{m}(t)$ a função de monitoração formada pelo concatenamento sucessivo da funções exponenciais (19) resultantes do esquema de controle adotado, para cada subintervalo $\Delta \tau_{i}$, em cada ciclo de busca de período $T_{s}$.

A Figura 2 ilustra o princípio de funcionamento da função de monitoração $\varphi_{m}(t)$ e o módulo do erro de rastreamento $|e(t)|$ (com notação simplificada). A cada chaveamento ocorre um salto de dimensão $\mu$, parâmetro que define a ordem das oscilações em torno de $x^{*}$, conforme será demonstrado mais adiante.

\subsection{Propriedades dinâmicas do sistema de controle}

Com a lei de controle (16)-(17), analisa-se a seguir o comportamento dinâmico do sistema para concluir sobre a convergência da busca extremal.

Primeiramente, supondo que o sinal de controle seja corretamente estimado desde o instante inicial da i-ésima busca direcional, tem-se $U=\operatorname{sgn}\left(k_{p_{i}}\right)$ e portanto em (13)



Figura 2. As trajetórias de $\varphi_{m}(t)$ e $|e(t)|$.

$$
\dot{e} \leq-\lambda e+\left|k_{p_{i}}\right|\left(-\rho \operatorname{sgn}(e)+\left|d_{e}\right|\right) \leq-\lambda e,
$$

Conclui-se assim que, enquanto o erro é "controlável" a lei de controle proposta reduz exponencialmente o erro de seguimento da rampa resultando em um aumento da função objetivo $h(x)$. Então, uma das possibilidades seguintes será realizada:

- $y=h(x)$ cresce até o final da i-ésima busca;

- y é levado à vizinhança de um máximo direcional, ao longo da i-ésima direção e o erro perde controlabilidade. Nesse caso, de maneira similar ao que ocorre em uma busca extremal escalar (Aminde et al. (2013) ou Oliveira et al. (2012), Apêndice C (ii)), o erro residual oscila com pequena amplitude de ordem $\mathcal{O}(\mu)$ em torno de um máximo direcional (não necessariamente único) até que nova busca direcional inicie;

- Se no início da busca, o erro já for não controlável (próximo a um extremo direcional), a função $h$ permanecerá dentro de um pequeno intervalo de variação de ordem $\mathcal{O}(\mu)$, como no item anterior.

Assim, qualquer que seja a situação, a função objetivo ou aumenta ou permanece praticamente constante na i-ésima busca.

Entretanto, se o sinal estimado for incorreto inicialmente, teríamos de (13) e (14):

$$
\dot{e}=+\left|k_{p_{i}}\right| \rho \operatorname{sgn}(e)-p,
$$

Multiplicando ambos os membros dessa equação por $\operatorname{sgn}(e)$, tem-se para $e \neq 0$ :

$$
\frac{d|e|}{d t}=+\rho\left|k_{p_{i}}\right|-p \operatorname{sgn}(e),
$$

o que implica que $|e(t)|$ cresce. Como a função de monitoração decresce e a velocidade de crescimento de $|e(t)|$ pode ser feita arbitrariamente grande aumentando-se $\delta$, o SW chaveará para o sinal correto de $k_{p_{i}}$ em tempo arbitrariamente curto. Assim conclui-se que se o período cíclico for suficientemente grande para que o tempo necessário para encontrar a direção de controle seja desprezível, passa-se logo para a mesma situação anterior, concluindo-se que também nesse caso, a busca leva a um aumento de $h$.

As seguintes Proposições são então concluídas.

Proposição 1. Considere o sistema (2)-(3), lei de controle (16 ), trajetória de referência (10), e direção de busca (11). Fora da região $\mathcal{D}_{\Delta_{i}}$, dada a direção de controle inicial arbitrária, pode-se escolher $\delta>0$ suficientemente grande tal que a direção de controle correta é encontrada no 
máximo em $\tau_{i}+\epsilon$, com $\epsilon$ arbitrariamente pequeno e menor do que o sub-intervalo $T_{s} / n$.

Proposição 2. Para $i$-ésima direção de busca ativa a função objetivo $h$ ou cresce ou permanece no mesmo valor, modulo pequenas oscilações que podem ser arbitrariamente reduzidas reduzindo-se o parâmetro $\Delta$.

\subsection{Convergência Global}

Nesta seção, é mostrado que o controlador cíclico multivariável por realimentação de saída baseado em função de monitoração conduz $x$ para a região $\mathcal{D}_{\Delta}$ onde se encontra o maximizador desconhecido $x^{*}$ definido em (H2). Isto não implica que $x$ permanece em torno de $\mathcal{D}_{\Delta}, \forall t$. Entretanto, as oscilações em torno de $y^{*}$ podem ser feitas da ordem $\mathcal{O}(\mu)$.

Teorema 1. Considere o sistema (2)-(3), lei de controle (8), trajetória de referência (10), direção de busca (11), função de monitoração (19)-(20) e função de modulação (17). Assuma que (H1) - (H2) sejam satisfeitas e que $T_{s}$, o período do ciclo de buscas direcionais, seja suficientemente grande. Então a região $\mathcal{D}_{\Delta}$ em $(\mathbf{H 2})$ é globalmente atrativa, sendo alcançada em tempo finito.

Prova: A prova é feita por absurdo. De acordo com as Proposições 1-2, a cada busca direcional a função objetivo cresce ou permanece quase constante. $\mathrm{O}$ período $T_{s}$ deve ser suficientemente grande para permitir que a direção de controle seja encontrada em cada busca direcional que comece com erro controlável.

Suponha-se por absurdo que $h(t)$, que é (essencialmente) não decrescente e necessariamente tende a um valor constante $h_{-}$modulo oscilações de amplitude $\varepsilon$ arbitrariamente pequenas, e que $h_{-}$esteja abaixo do ínfimo $h_{-}^{*}$ de $h$ em $\mathcal{D}_{\Delta}$. Mais precisamente, suponha que $h_{-}^{*}>h_{-}+\varepsilon$. Como na busca cíclica, pelo menos em alguma direção, o erro $e$ é controlável, $h(t)$ necessariamente cresce além de $h_{-}+\varepsilon$, contradizendo a hipótese. Assim, a região $\mathcal{D}_{\Delta}$ em $(\mathbf{H 2})$ é globalmente atrativa, sendo alcançada em tempo finito.

\section{EXEMPLO ILUSTRATIVO}

Como forma de exemplo, considere uma planta cuja função objetivo seja desconhecida, e dinâmica de simples integrador descrita por

$$
\begin{aligned}
& \dot{x}=v \\
& y=-\left(x_{1}^{2}+\left(x_{2}-x_{1}^{2}\right)^{2}\right)
\end{aligned}
$$

Analisando a função objetivo (27), conclui-se que os parâmetros ótimos desejados são $x^{*}=(0,0)$ e $y^{*}=0$ e tem a forma ilustrada na Figura 6. Esta função é conhecida na literatura como função de Rosenbrock, ou função banana. Note que as curvas de nível delimitam domínios nãoconvexos.

As simulações foram realizadas considerando os seguintes parâmetros: $p=1, r_{0}=-5, \rho(t)=5, \lambda=1, \delta=0.1$, $T_{s}=5 s$ e $\mu=0.2$.

A Figura 3 ilustra o resultado do objetivo de controle, a busca pelo vetor otimizador $x^{*}=(0,0)$ e respectiva saída $y^{*}=0$. Note também que as oscilações em torno de $x^{*}$ são limitadas à ordem $\mathcal{O}(\mu)$ (ver detalhe do gráfico Figura $3(\mathrm{~b})$ ). A função objetivo, apresentada na Figura 6, pode representar um mapeamento de otimização de potência de uma turbina eólica, onde o vetor $x$ representaria torque e o ângulo da turbina (Creaby et al., 2009). Esta figura mostra a busca em cada direção partindo de duas condições iniciais, $x_{0}=(-1.5,1.5)$ e $x_{0}=(1.5,-1.5)$.
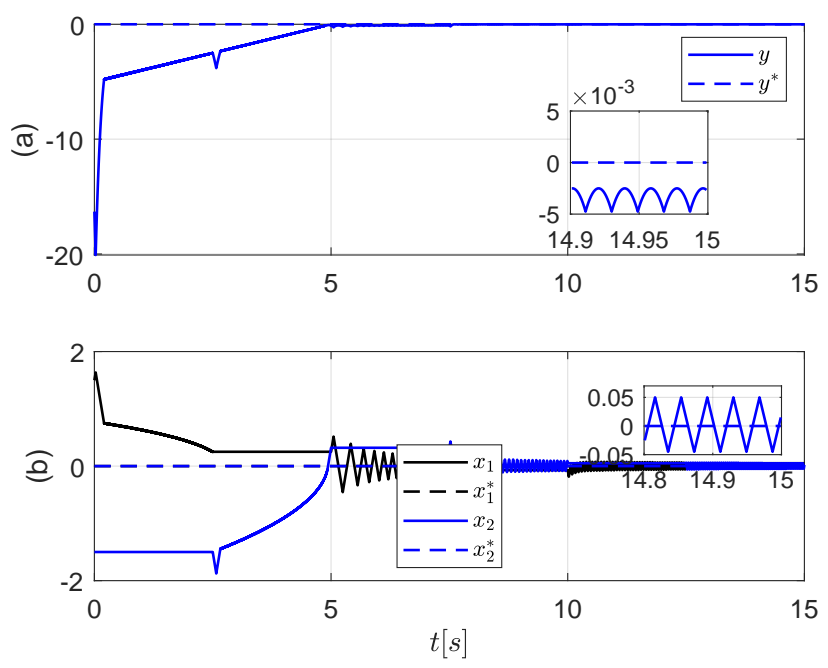

Figura 3. (a) A saída da planta converge para $y^{*}=0$ e (b) parâmetros do vetor $x$ convergem para $x^{*}=(0,0)$ partindo da condição inicial $x(0)=(1.5,-1.5)$.

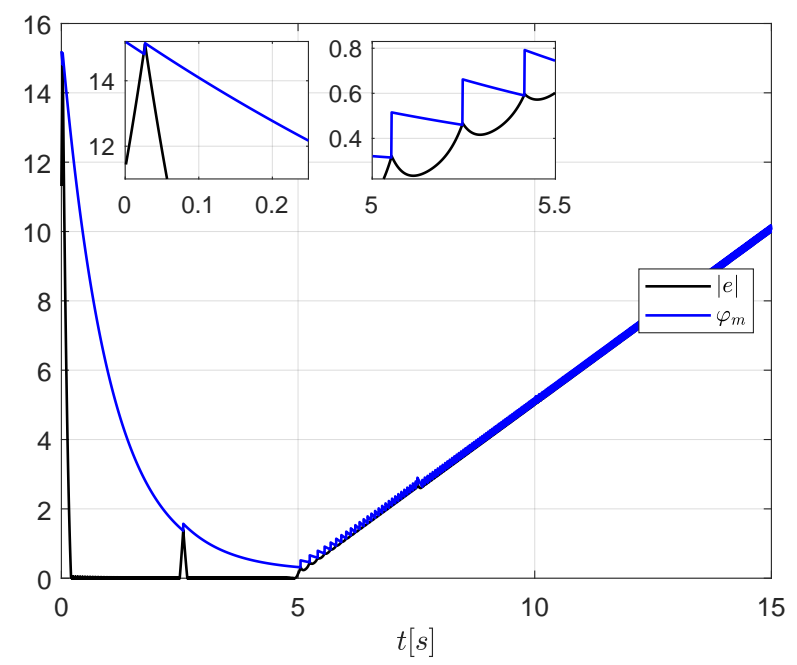

Figura 4. Função de monitoração $\varphi_{m}$ e o valor absoluto do erros $|e|$.

Por sua vez a Figura 4 ilustra o comportamento da função de monitoração $\varphi_{m}$ ante a norma de erro $|e|$. Como pode-se observar nos destaques desta figura, o destaque à esquerda mostra que a estimativa de direção de controle é errônea e por isso o erro começa a crescer e logo a direção é acertada e o erro decresce, o destaque à direita mostra que a cada instante de mudança da direção de controle ocorre um salto $\mu=0.2$ conforme a definição da função de monitoração. Após $5 s$, instante em que chega-se a $x^{*}$ começa o chaveamento indeterminado causado pela falta 
de controlabilidade, sobre a trajetória com inclinação de $r(t)$.

Os dois sinais de controle $u_{1}$ e $u_{2}$ estão ilustrados na Figura 5a. Nela observa-se que a amplitude de chaveamento é definida pela função de modulação $\rho(t)=5$. Além disso, é notório o chaveamento em alta frequência que poderia causar o fenômeno indesejado denominado "chattering". Contudo, a função objetivo (3) recebe os sinais de controle filtrados $x$. Enquanto a Figura 5b ilustra a troca de direção de busca, efeito do controle cíclico, entre $\sigma_{1}$ e $\sigma_{2}$. É notorio que o período é de $5 s$ e ocorrem dois ciclos, portanto, $\Delta \tau_{i}=2.5 \mathrm{~s}$.
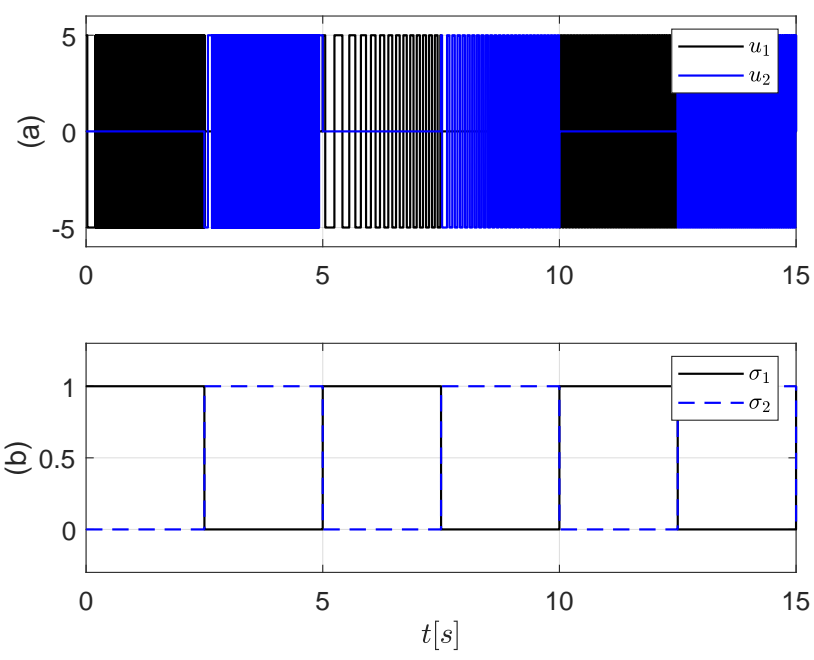

Figura 5. (a) Sinais de controle $u_{1}$ e $u_{2}$ e (b) troca de direção de busca cíclica, com período de $T s=5 \mathrm{~s}$.

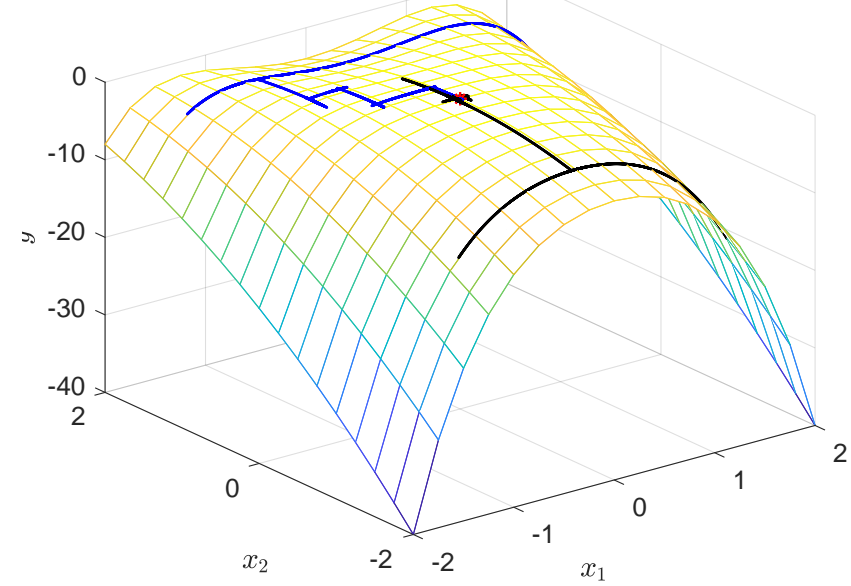

Figura 6. Simulação numérica para duas condições iniciais $x_{0}=(-1.5,1.5)$ e $x_{0}=(1.5,-1.5)$ sobre a superfície da função objetivo $y=h(x)$ cujos estados convergem para origem.

A Figura 7 ilustra o rastreamento da trajetória de referência. Através dela, confirma-se a teoria descrita no início da Seção 3, a saída rastrea a referência até encontrar $y^{*}=0$ e permanece nele.

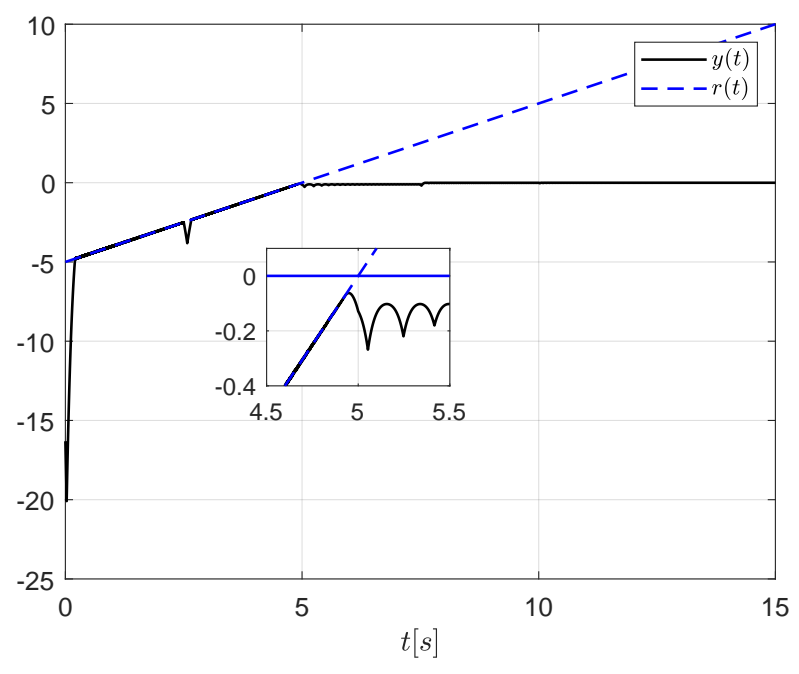

Figura 7. A saída $y$ rastreia o modelo de referência até sua estagnação no ponto máximo, $y^{*}=0$.

A Figura 8 ilustra o resultado de simulação usando estratégia de controle proposta por Salamah and Özguner (2018). Um breve comparativo ao nosso resultado (ver Figura 3), nota-se que o algoritmo aqui proposto tem convergência relativamente mais rápida, apesar de se usar mesma função de modulação, ilustrada na Figura 9a) e mesma condição inicial $x(0)=(-1.5,1.5)$ e mesmo tempo de busca por cada direção, $\Delta \tau_{i}=2.5 \mathrm{~s}$.
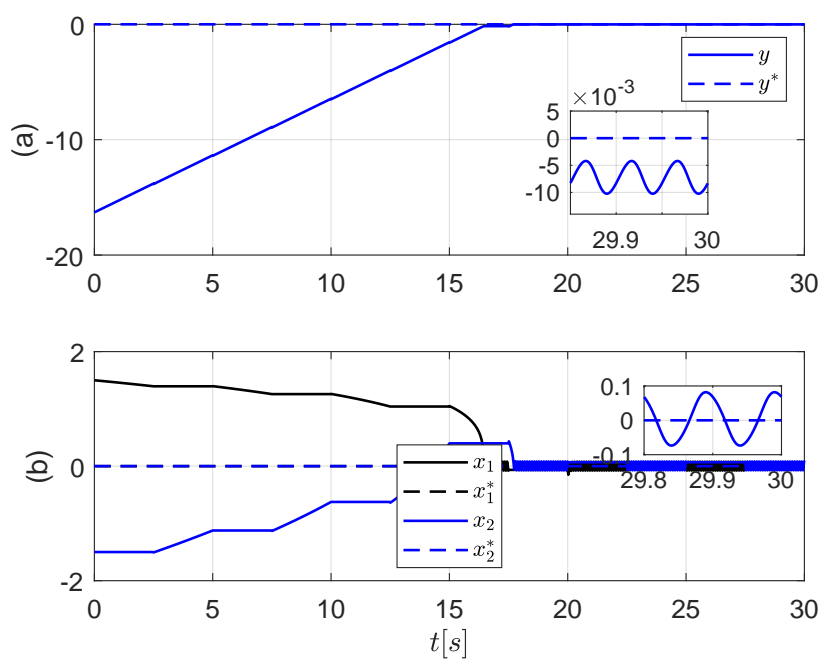

Figura 8. Parâmetros do vetor $x$ convergem para origem usando estratégia de controle de Salamah and Özguner, 2018 .

\section{CONCLUSÕES}

Um controlador extremal cíclico multivariável baseado em função de monitoração foi desenvolvido para uma classe de plantas estáticas multivariáveis não lineares. Supõe-se que tal função multivariável tem um único extremo máximo. A abordagem resultante garante convergência global da saída do sistema para uma pequena vizinhança do extremo usando-se apenas realimentação da saída, i.e., o valor da 



Figura 9. Sinais de controle $u_{1}$ e $u_{2}$ e troca de direção de busca, usando estratégia de controle de Salamah and Özguner, 2018.

função objetivo. Resultados numéricos consistentes de otimização multivariável para o sinal de saída foram apresentados, ilustrando o desempenho do controlador proposto. Uma simulação comparativa mostra a maior rapidez de convergência em relação a Salamah and Özguner (2018). Uma extensão para plantas com dinâmicas não-lineares em série com o mapeamento estático multivariável está sendo pesquisada.

\section{REFERÊNCIAS}

Aminde, N.O., Oliveira, T.R., , and Hsu, L. (2018). Controle extremal multivariável via funções de monitoração. XXII Congresso Brasileiro de Automática ( $C B A)$.

Aminde, N.O., Oliveira, T.R., and Hsu, L. (2013). Global output-feedback extremum seeking control via monitoring functions. 52nd IEEE Conference on Decision and Control (CDC), 1031-1036.

Ariyur, K.B. and Krstić, M. (2002). Analysis and design of multivariable extremum seeking. American Control Conference, 2903 - 2908.

Ariyur, K.B. and Krstić, M. (2003). Real-Time Optimization by Extremum-Seeking Control. John Wiley \& Sons.

Calli, B., Caarls, W., Jonker, P., and Wisse, M. (2012). Comparison of extremum seeking control algorithms for robotic applications. International Conference on Intelligent Robots and Systems, 3195-3202.

Creaby, J., Li, Y., and Seem, J.E. (2009). Maximizing wind turbine energy capture using multivariable extremum seeking control. Wind Engineering.

Filippov, A.F. (1964). Differential equations with discontinuous right-hand side. American Math. Soc. Translations, 42(2), 199-231.

Ghaffari, A., Krstic, M., and Nesic, D. (2012). Multivariable newton-based extremum seeking. Automatica, 1759 1767.

Hsu, L., Oliveira, T.R., and Cunha, J.P.V.S. (2014). Extremum seeking control via monitoring function and timescaling for plants of arbitrary relative degree. 13th IEEE Workshop on Variable Structure Systems, 1-6.
Krstić, M. and Wang, H.H. (2000). Stability of extremum seeking feedback for general nonlinear dynamic systems. Automatica, 595-601.

Oliveira, T.R., Hsu, L., and Peixoto, A.J. (2011). Outputfeedback global tracking for unknown control direction plants with application to extremum-seeking control. Automatica, 47, 2029-2038.

Oliveira, T.R., Aminde, N.O., and Hsu, L. (2014). Monitoring function based extremum seeking control for uncertain relative degrees with light source seeking experiments. 53rd IEEE Conference on Decision and Control $(C D C), 3456-3462$.

Oliveira, T.R., Peixoto, A.J., and Hsu, L. (2010). Sliding mode control of uncertain multivariable nonlinear systems with unknown control direction via switching and monitoring function. IEEE Trans. Automat. Contr., 55(4), 1028-1034.

Oliveira, T.R., Peixoto, A.J., and Hsu, L. (2012). Global real-time optimization by output-feedback extremumseeking control with sliding modes. Journal of Franklin Institute, 349, 1397-1415.

Oliveira, T.R., Peixoto, A.J., Nunes, E.V.L., and Hsu, L. (2007). Control of uncertain nonlinear systems with arbitrary relative degree and unknown control direction using sliding modes. Int. J. Adapt. Control Signal Process, 21, 692-707.

Rotea, M.A. (2000). Analysis of multivariable extremum seeking algorithms. American Control Conference.

Salamah, Y.B. and Özguner, U. (2018). Sliding mode multivariable extremum seeking control with application to wind farm power optimization. American Control Conference (ACC), 5321 - 5326.

Tan, Y., Moase, W., Manzie, C., Nesić, D., and Mareels, I. (2010). Extremum seeking from 1922 to 2010. 29th Control Conference Chinese (CCC), 14-26.

Toloue, S.F. and Moallem, M. (2016). Multivariable sliding-mode extremum seeking control with application to alternator maximum power point tracking. IECON 2016 - 42nd Annual Conference of the IEEE Industrial Electronics Society.

Toloue, S.F. and Moallem, M. (2017). Multivariable sliding-mode extremum seeking control with application to alternator maximum power point tracking. TRANSACTIONS ON INDUSTRIAL ELECTRONICS.

Xiao, Y., Li, Y., and Seem, J.E. (2014). Multi-variable extremum seeking control for mini-split air-conditioning system. International Refrigeration and Air Conditioning Conference.

Yaghoubi, S., Dehghani, M., and Khayatian, M. (2018). Adaptive backstepping extremum seeking control of a class of nonlinear systems. Iran J Sci Technol Trans Mech Eng, 1 - 9. doi:https://doi.org/10.1007/ s40997-018-0167-8.

Yan, L., Hsu, L., Costa, R.R., and Lizarralde, F. (2003). Variable structure model reference adaptive control for systems with unknown high frequency gain. Conference on Decision and Control.

Yan, L., Hsu, L., and Xiuxia, S. (2006). A variable structure mrac with expected transient and steady-state performance. Automatica. 